\title{
Glycosylation of natriuretic peptides in obese heart failure: mechanistic insights
}

\author{
Vibhu Parcha ${ }^{1}$, Pankaj Arora ${ }^{1,2}$ \\ ${ }^{1}$ Division of Cardiovascular Disease, Department of Medicine, University of Alabama at Birmingham, Birmingham, AL, USA; ${ }^{2}$ Section of \\ Cardiology, Birmingham Veterans Affairs Medical Center, Birmingham, AL, USA \\ Correspondence to: Pankaj Arora, MD, FAHA. Division of Cardiovascular Disease, Department of Medicine, 1670 University Boulevard, Volker Hall \\ B140, University of Alabama at Birmingham, Birmingham, AL 35294-0019, USA. Email: parora@uabmc.edu. \\ Provenance: This is an invited article commissioned by our guest section editor Dr. Ying Zhao (Department of Laboratory Medicine, the First \\ Affiliated Hospital, College of Medicine, Zhejiang University, China). \\ Comment on: Lewis LK, Raudsepp SD, Prickett TCR, et al. ProBNP that is not glycosylated at threonine 71 is decreased with obesity in patients with \\ heart failure. Clin Chem 2019;65:1115-24.
}

Submitted Aug 30, 2019. Accepted for publication Oct 11, 2019.

doi: $10.21037 /$ atm.2019.10.59

View this article at: http://dx.doi.org/10.21037/atm.2019.10.59

Natriuretic peptides (NPs) are cardiac derived hormones that are secreted in response to increased wall stress, stretching of the myocardium, volume overload, and angiotensin II stimulation. The principal circulating NPs, atrial and B-type NP (ANP and BNP respectively) are released as precursor molecules (proANP and proBNP) which are processed to active components ANP and BNP, and inactive amino-terminal fragments NT-proANP and NT-proBNP (1). Mature NPs bind and activate membrane receptors called NP receptor A and B (signaling receptor), which results in increased glomerular filtration, sodium excretion, vasodilation, and direct inhibition of the reninangiotensin-aldosterone system. Mature NPs are removed from the circulation by proteolysis by an enzyme called neprilysin and via NP receptor C (clearance receptor) (2). In states of volume and pressure overload such as heart failure, there is an increased concentration of NPs in circulation, which has led to their use as diagnostic and prognostic markers for heart failure. BNP and its related peptides have greater in-vitro stability and better diagnostic utility compared to ANP. Therefore, BNP and its fragments (proBNP and NT-proBNP) emerged as ideal candidates for assessment and subsequently became established as gold-standard biomarkers for the risk assessment, diagnosis, prognostication, and treatment monitoring of heart failure $(3,4)$.

\section{Obesity and NPs}

Under normal physiological conditions, biological variability of NP levels has been described with body mass index (BMI) (5), in addition to age, sex (5), race (5-7), and glomerular filtration rate (5) contributing to the inter- and intra-individual differences in NP levels. This variability persists in the disease state of heart failure. Several population-based studies have shown that there is an inverse association of BMI with NP levels $(8,9)$, with obese individuals having $30-40 \%$ lower NP levels compared with lean individuals. Obesity is well recognized as a NP deficient state (5) and an important contributor to the etiopathogenesis of heart failure. Accurate clinical assessment of heart failure is difficult in obese individuals $(10,11)$ which underlines the significance of accurately measuring these biomarkers using appropriate assays, as demonstrated by Lewis et al. (12).

\section{ProBNP not glycosylated at Thr71 (NG-Thr71) is a surrogate for proBNP processing}

In response to stimulus, the $\mathrm{BNP}$ gene $(N P P B)$ is transcribed and translated into 134-amino acid pre-proBNP, which is converted to prohormone (proBNP 1-108). After synthesis, proBNP undergoes post-translational modification before being released into the circulation. The 


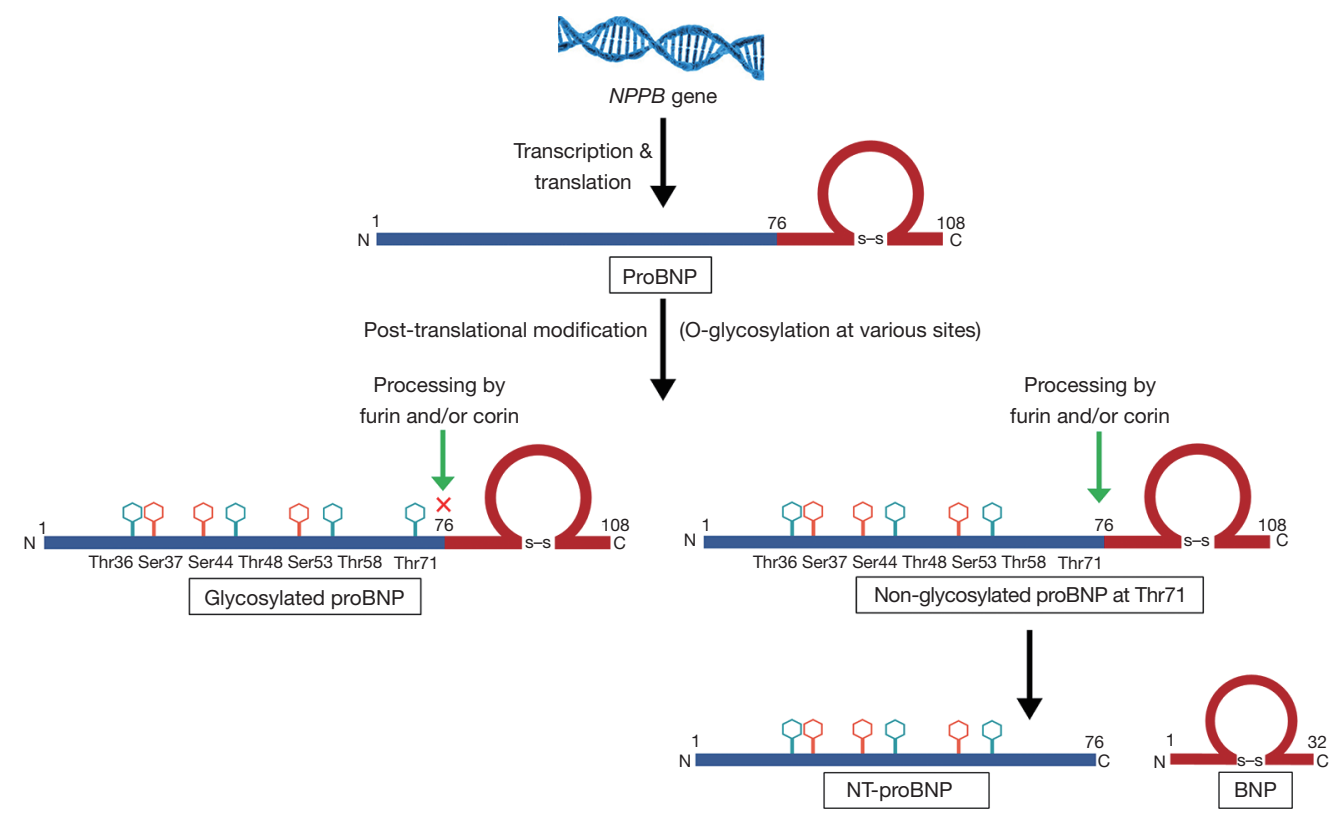

Figure 1 Schematic representation of proBNP processing. BNP, B-type natriuretic peptide.

proBNP molecule undergoes extensive O-glycosylation which has been described at Thr36, Ser37, Ser44, Thr48, Ser53, Thr58 and Thr71 sites of the peptide $(13,14)$. The proBNP molecule usually undergoes endoproteolytic cleavage by furin and/or corin, between Arg76 and Ser77 amino-acids positions to yield inactive NT-proBNP 1-72 and mature BNP 1-32 (Figure 1). A mixture of the active BNP 1-32 and its proteolytic fragments are present in the circulation. A combination of multiple peptide fragments is typically measured and reported by the commercially available immunoassays (15). Prior work using mass spectrometry-based assays suggests that there is very little bioactive BNP moiety present in the circulation of patients with heart failure (16). In addition to the proteolytic fragments, proBNP is abundant in the circulation of heart failure patients and is a major contributor to BNPimmunoreactivity in plasma (17) as measured by the various commercially available assays. Glycosylation in the region close to the cleavage site increases the stability of proBNP by preventing furin and/or corin enzyme-mediated processing (Figure 1). The enzymatic processing of proBNP is especially suppressed by $\mathrm{O}$-glycosylation at Thr71 which has been reported from $20 \%$ to $70 \%(18,19)$. Combination of the dysregulation in the processing of proBNP into active $\mathrm{BNP}$ and proteolytic breakdown of biologically active BNP into inactive fragments may explain the low BNP observed in the heart failure state in prior work done using the mass spectrometry-based assays (15). Additionally, the increased glycosylation and impaired processing may explain the lower NP levels in the relative NP deficient states like obesity (5) and African-American race (6). To summarize, by being able to measure the various non-glycosylated forms of proBNP, one can untangle the reasons for the relative difference in NP levels in NP deficient subgroups.

\section{Limitations of the commercially available BNP and NT-proBNP immunoassays}

Multiple commercial immunoassays are available for the measurement of BNP and NT-proBNP levels with a large between-method variability for BNP and NTproBNP (20-22). Though proBNP has little physiological activity, its additional presence in plasma causes crossreactivity in the commercially available assays for BNP and NT-proBNP. Most BNP assays exhibit cross-reactivity with proBNP because of the presence of mature BNP ring structure within proBNP peptide. Glycosylation at various sites provides steric hindrance for the binding of the epitope of the antibodies used by various assays for measurement of NT-proBNP. The only FDA-approved and commercially 


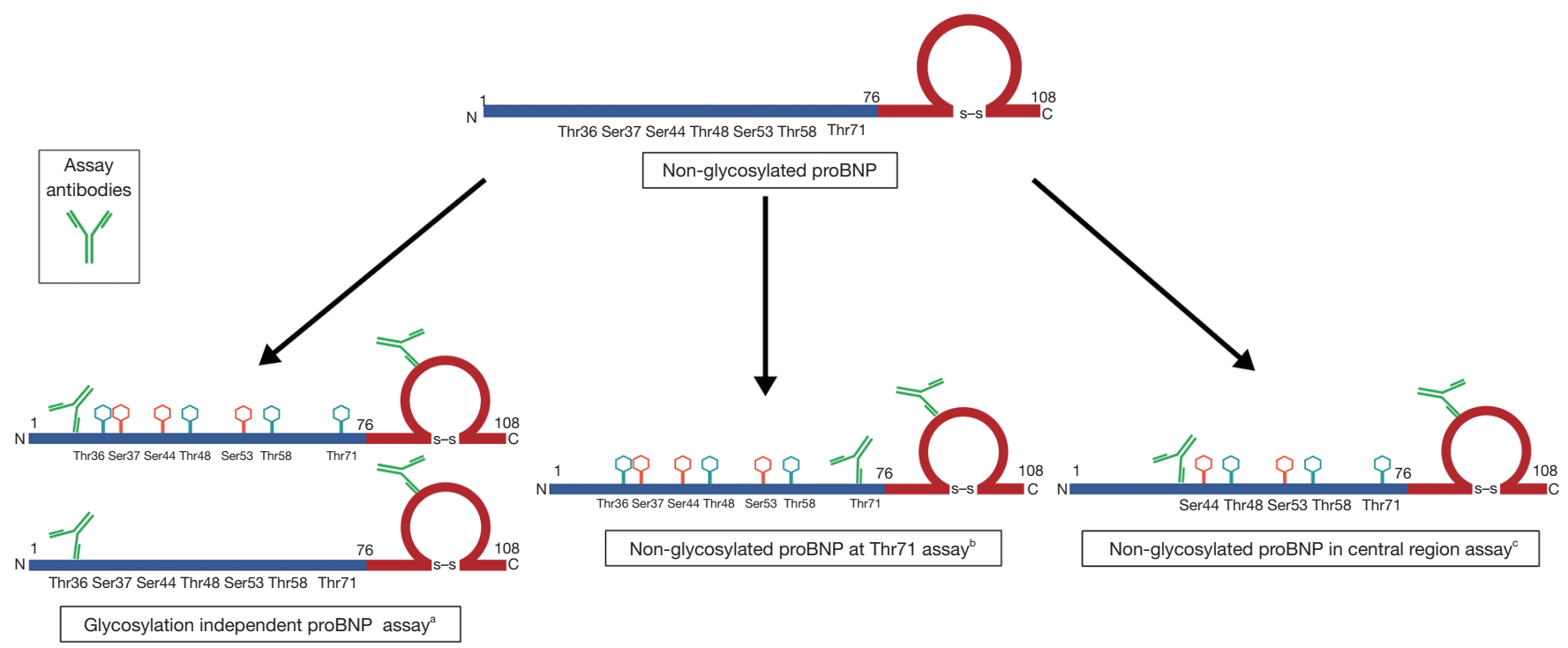

Figure 2 Schematic representation of assay detection of non-glycosylated variants of proBNP. a, assay antibodies are not sensitive to glycosylation status and capture all proBNP variants independent of glycosylation status; ${ }^{\mathrm{b}}$, assay antibodies are sensitive to glycosylation at threonine 71 position and capture only non-glycosylated at threonine 71 variant of proBNP; ${ }^{c}$, assay antibodies are sensitive to glycosylation in the central region (proBNP 31-39 region) and capture only non-glycosylated in central region variants of proBNP. BNP, B-type natriuretic peptide.

available NT-proBNP assay contains antibodies that bind to the central region of proBNP, which is more likely to be glycosylated and restricts the binding of the antibodies (21). It has been shown that up to $80 \%$ of the circulating proBNP maybe glycosylated in the central region (23). Since this assay and other NT-proBNP assays have antibodies which can bind only to the non-glycosylated peptide fragments, the assays assess only a sub-fraction of the total concentration. Mass spectrometry method to measure the various glycosylated and non-glycosylated NPs has been developed to overcome the limitations of the immunoassays and has promising potential (15).

\section{ProBNP NG-Thr71 in obese heart failure patients}

In the study by Lewis et al. (12), the authors developed immunoassays specific for the measurement of proBNP NG-Thr71, proBNP not centrally glycosylated (NG-C) and total proBNP (non-glycosylated and glycosylated) among obese heart failure patients. The assay used two sets of antibodies, one of which was specific for the ring structure of the peptide, and the other set was specific for the
$\mathrm{N}$-terminal portion of the peptide (Figure 2). In detecting the total proBNP, they used the antibodies which would bind away from the central region and thus would bind to all proBNP molecules irrespective of the glycosylation status (Figure 2). To detect the proBNP molecules that were NG-Thr71, the assay used antibodies which were specific for the Thr71 region of the peptide. Finally, to detect the non-centrally glycosylated form of proBNP, they used antibodies that would bind to the central region of the peptide (Figure 2). The assays developed by the authors had an intra-assay $(<10 \%)$ and the inter-assay coefficient of variance ranging from $10-15 \%$. The lower limit of detection for their assay was 18,18 , and $21 \mathrm{ng} / \mathrm{L}$ for total proBNP, NG-Thr71, and NG-C, respectively (12). The authors determined that in patients with heart failure, the levels of total proBNP was highest, followed by NGThr71 form, and the lowest for NG-C (12). They were able to calculate that $\sim 35 \%$ of the proBNP peptides were glycosylated at Thr71 and $\sim 83 \%$ proBNP peptides were glycosylated centrally. Using the approach identified by the authors (12), one can determine proBNP peptides with sitespecific glycosylation and calculate the ratios of glycosylated 
and non-glycosylated proBNP variants. This proves to be an exciting prospect for the field which has recently been ignited again with the effect of NP augmenting medications on commercially available immunoassays (24).

\section{Obesity and proBNP glycosylation}

The authors studied the levels of the various forms of the peptide in the NP deficient population of obese individuals with heart failure and healthy controls. Lower NG-Thr71 and NT-proBNP plasma concentrations were observed to be associated with higher BMI, while the total proBNP remained stable across BMI. ProBNP NGThr71 is processed into the biologically active BNP 1-32, which shows the beneficial biological effects of NPs in maintaining cardiovascular homeostasis. Since glycosylation at Thr71 reduces the production of bioactive BNP 1-32, having lower levels of NG-Thr71 may be one of the plausible explanations behind lower levels of BNP and NTproBNP described in obese individuals. Previous studies have described differential rates of proBNP processing with different disease states (19). Results from Lewis et al. add to the previous findings and support the hypothesis of differential glycosylation and processing of proBNP, especially in sub-populations like obese heart failure patients, who have relatively lower NP levels. By replicating promising work like this on a larger scale, one can determine which specific glycosylated and non-glycosylated forms are associated with a particular population subgroup. Additionally, by measuring these forms in NP deficient healthy population, conceivable explanations for the relative NP deficiency may be derived based on variability in glycosylation driven processing of proBNP peptide.

\section{Glycosylation of proBNP and sacubitril/valsartan}

Rapid and easy measurement of the various glycosylated and non-glycosylated forms of NPs has gained importance since the introduction of sacubitril/valsartan, the first in the class of angiotensin receptor-neprilysin inhibitors (ARNIs). Sacubitril/valsartan inhibits neprilysin enzyme which breaks down BNP and is associated with an increase in measured BNP levels and decrease in NT-proBNP levels of heart failure patients (25). Sacubitril/valsartan has shown to increase the levels of proBNP glycosylated at Thr71, which would imply decreased processing of proBNP into BNP and NT-proBNP (24). Additionally, since proBNP strongly cross-reacts with BNP assays independent of glycosylation, the observed increase in BNP levels in ARNI treated patients could be possibly explained by the increased production of non-cleavable glycosylated variants of proBNP. By using the assays developed by Lewis et al. NG-Thr71 proBNP may be measured in response to ARNI therapy to evaluate the role of glycosylation in different heart failure phenotypes. Evaluating the levels of NG-Thr71 in response to sacubitril/valsartan treatment in subgroups with NP deficiency may provide additional mechanistic insights. In the era of NP augmenting medication, the ability to detect the relevant forms of NP becomes pertinent to derive the accurate diagnostic and prognostic conclusions from these heart failure biomarkers.

The exciting possibilities suggested by this important work by Lewis et al. (12) is accompanied by certain limitations such as it being a single-center study with a limited population, lacking description about the race of the individuals and the etiology of heart failure. Since circulating NP levels vary by sex (5), the findings of the study could be strengthened by evaluating the sex-related differences in the measured non-glycosylated proBNP variants. Additionally, having a comparator group in the form of healthy obese individuals would have been insightful in understanding if the difference in NG-Thr71 is generalizable to the entire obese phenotype independent of heart failure status. Furthermore, the decreased levels of the proBNP variant observed in the obese sub-group could also be accounted for by post-translational modifications of proBNP other than O-glycosylation. The authors found the prognostic value of NG-Thr71 variant in the study cohort to be inferior to the commercially available NT-proBNP immunoassay, which may be due to small size of the cohort. Larger studies measuring various forms of proBNP peptides in a larger, multi-center, diverse cohort, with a comparator healthy population, are needed to help further the knowledge and understanding of NP pathophysiology.

In conclusion, the study by Lewis et al. (12) supplements the knowledge about the complex biochemistry of the proBNP and elaborates upon the intricacies of the NP physiology. Differences in glycosylation could be a feasible explanation for the lower NP levels in obese patients. Measurement of a single biomarker variant may not be sufficient to draw accurate diagnostic and prognostic inferences, particularly in an era of contemporary heart failure therapy with ARNIs as class I medication. Moreover, further validation of the proBNP assays is needed to determine the clinically useful BNP variant. Advancing the understanding of the NP physiology and pharmacology 
depends upon standardizing NP assays to measure the glycosylated states of proBNP. Further work is needed to get a comprehensive understanding of the complex puzzle to aid the ever-expanding realm of NPs as biomarkers.

\section{Acknowledgments}

Funding: Dr. Pankaj Arora is supported by National Institutes of Health Mentored Patient-Oriented Research Award 1K23HL146887-01.

\section{Footnote}

Conflicts of Interest: The authors have no conflicts of interest to declare.

Ethical Statement: The authors are accountable for all aspects of the work in ensuring that questions related to the accuracy or integrity of any part of the work are appropriately investigated and resolved.

\section{References}

1. Mangiafico S, Costello-Boerrigter LC, Andersen IA, et al. Neutral endopeptidase inhibition and the natriuretic peptide system: an evolving strategy in cardiovascular therapeutics. Eur Heart J 2013;34:886-93c.

2. Potter LR. Natriuretic peptide metabolism, clearance and degradation. FEBS J 2011;278:1808-17.

3. Yancy CW, Jessup M, Bozkurt B, et al. 2017 ACC/ AHA/HFSA focused update of the 2013 ACCF/AHA guideline for the management of heart failure: a report of the American College of Cardiology/American Heart Association Task Force on Clinical Practice Guidelines and the Heart Failure Society of America. J Am Coll Cardiol 2017;70:776-803.

4. Patel N, Cushman M, Gutiérrez OM, et al. Racial differences in the association of NT-proBNP with risk of incident heart failure in REGARDS. JCI Insight 2019. doi: 10.1172/jci.insight.129979.

5. Patel N, Gutiérrez OM, Arora G, et al. Race-based demographic, anthropometric and clinical correlates of N-terminal-pro B-type natriuretic peptide. Int J Cardiol 2019;286:145-51.

6. Bajaj NS, Gutiérrez OM, Arora G, et al. Racial differences in plasma levels of $\mathrm{N}$-terminal pro-B-type natriuretic peptide and outcomes: the reasons for geographic and racial differences in stroke (REGARDS) study. JAMA
Cardiol 2018;3:11-7.

7. Patel N, Russell GK, Musunuru K, et al. Race, natriuretic peptides, and high-carbohydrate challenge: a clinical trial. Circ Res 2019. [Epub ahead of print].

8. Wang TJ, Larson MG, Levy D, et al. Impact of obesity on plasma natriuretic peptide levels. Circulation 2004;109:594-600.

9. Das SR, Drazner MH, Dries DL, et al. Impact of body mass and body composition on circulating levels of natriuretic peptides: results from the Dallas Heart Study. Circulation 2005;112:2163-8.

10. Maisel AS, Krishnaswamy P, Nowak RM, et al. Rapid measurement of B-type natriuretic peptide in the emergency diagnosis of heart failure. N Engl J Med 2002;347:161-7.

11. Daniels LB, Clopton P, Bhalla V, et al. How obesity affects the cut-points for B-type natriuretic peptide in the diagnosis of acute heart failure. Results from the breathing not properly multinational study. Am Heart J 2006;151:999-1005.

12. Lewis LK, Raudsepp SD, Prickett TCR, et al. ProBNP that is not glycosylated at threonine 71 is decreased with obesity in patients with heart failure. Clin Chem 2019;65:1115-24.

13. Schellenberger U, O'Rear J, Guzzetta A, et al. The precursor to B-type natriuretic peptide is an O-linked glycoprotein. Arch Biochem Biophys 2006;451:160-6.

14. Seferian KR, Tamm NN, Semenov AG, et al. Immunodetection of glycosylated NT-proBNP circulating in human blood. Clin Chem 2008;54:866-73.

15. Miller WL, Phelps MA, Wood CM, et al. Comparison of mass spectrometry and clinical assay measurements of circulating fragments of B-type natriuretic peptide in patients with chronic heart failure. Circ Heart Fail 2011;4:355-60.

16. Niederkofler EE, Kiernan UA, O'Rear J, et al. Detection of endogenous B-type natriuretic peptide at very low concentrations in patients with heart failure. Circ Heart Fail 2008;1:258-64.

17. Luckenbill KN, Christenson RH, Jaffe AS, et al. Cross-reactivity of BNP, NT-proBNP, and proBNP in commercial BNP and NT-proBNP assays: preliminary observations from the IFCC Committee for Standardization of Markers of Cardiac Damage. Clin Chem 2008;54:619-21.

18. Semenov AG, Tamm NN, Seferian KR, et al. Processing of pro-B-type natriuretic peptide: furin and corin as candidate convertases. Clin Chem 2010;56:1166-76. 
19. Vodovar N, Séronde MF, Laribi S, et al. Post-translational modifications enhance NT-proBNP and BNP production in acute decompensated heart failure. Eur Heart J 2014;35:3434-41.

20. Clerico A, Zaninotto M, Prontera C, et al. State of the art of BNP and NT-proBNP immunoassays: the CardioOrmoCheck study. Clin Chim Acta 2012;414:112-9.

21. Semenov AG, Feygina EE. Standardization of BNP and NT-proBNP immunoassays in light of the diverse and complex nature of circulating BNP-related peptides. Adv Clin Chem 2018;85:1-30.

22. Semenov AG, Tamm NN, Apple FS, et al. Searching for a BNP standard: Glycosylated proBNP as a common

Cite this article as: Parcha V, Arora P. Glycosylation of natriuretic peptides in obese heart failure: mechanistic insights. Ann Transl Med 2019;7(22):611. doi: 10.21037/atm.2019.10.59 calibrator enables improved comparability of commercial BNP immunoassays. Clin Biochem 2017;50:181-5.

23. Nishikimi T, Ikeda $M$, Takeda $Y$, et al. The effect of glycosylation on plasma N-terminal proBNP-76 levels in patients with heart or renal failure. Heart 2012;98:152-61.

24. Nougué H, Pezel T, Picard F, et al. Effects of sacubitril/ valsartan on neprilysin targets and the metabolism of natriuretic peptides in chronic heart failure: a mechanistic clinical study. Eur J Heart Fail 2019;21:598-605.

25. McMurray JJ, Packer M, Desai AS, et al. Angiotensinneprilysin inhibition versus enalapril in heart failure. $\mathrm{N}$ Engl J Med 2014;371:993-1004. 